\title{
Molibresib Besylate
}

National Cancer Institute

\section{Source}

National Cancer Institute. Molibresib Besylate. NCI Thesaurus. Code C159943.

The besylate salt of molibresib, a small molecule inhibitor of the BET (Bromodomain and Extra-Terminal) family of bromodomain-containing proteins with potential antineoplastic activity. Upon administration, molibresib binds to the acetylated lysine recognition motifs on the bromodomain of BET proteins, thereby preventing the interaction between the BET proteins and acetylated histone peptides. This disrupts chromatin remodeling and gene expression. Prevention of the expression of certain growth-promoting genes may lead to an inhibition of tumor cell growth. Characterized by a tandem repeat of bromodomain at the $\mathrm{N}$-terminus, BET proteins, comprising of BRD2, BRD3, BRD4 and BRDT, are transcriptional regulators that play an important role during development and cellular growth. 